Article

\title{
Linking Man and Nature: Relictual Forest Coenosis with Laurus nobilis L. and Celtis australis L. in Antica Lavinium, Italy
}

\author{
Giulia Albani Rocchetti, Flavia Bartoli * (D), Emanuela Cicinelli, Fernando Lucchese and Giulia Caneva \\ Department of Science, University Roma Tre, Viale Marconi 446, 00146 Rome, Italy; \\ giulia.albanirocchetti@uniroma3.it (G.A.R.); emanuela.cicinelli@uniroma3.it (E.C.); \\ fernando.lucchese@uniroma3.it (F.L.); giulia.caneva@uniroma3.it (G.C.) \\ * Correspondence: flavia.bartoli@uniroma3.it
}

check for

updates

Citation: Albani Rocchetti, G.;

Bartoli, F.; Cicinelli, E.; Lucchese, F.; Caneva, G. Linking Man and Nature: Relictual Forest Coenosis with Laurus nobilis L. and Celtis australis L. in Antica Lavinium, Italy. Sustainability 2022, 14, 56. https://doi.org/ $10.3390 /$ su14010056

Academic Editor: Sharif Ahmed Mukul

Received: 5 November 2021

Accepted: 17 December 2021

Published: 21 December 2021

Publisher's Note: MDPI stays neutral with regard to jurisdictional claims in published maps and institutional affiliations.

Copyright: (c) 2021 by the authors Licensee MDPI, Basel, Switzerland. This article is an open access article distributed under the terms and conditions of the Creative Commons Attribution (CC BY) license (https:// creativecommons.org/licenses/by/ $4.0 /)$.

\begin{abstract}
The Mediterranean basin has been a refugium for relict plant taxa and native laurophyllic forests. The Latium coasts and, especially, the Antica Lavinium site, host relict forest communities, whose natural importance is enriched by their cultural value. Here, we aim at investigating the ecological framework, cultural and historical values, and management over time, of relict communities that have Laurus nobilis and Celtis australis as their priority habitats. To achieve this, we performed vegetation surveys and we conducted statistical analyses (PCA, NMDS). Among the 45 vegetation surveys, 25 were characterized by the two target species. The PCA analysis highlighted how the L. nobilis formations and the mixed formations with $C$. australis present some differences but are not sufficient to describe different coenosis. The comparison among similar forests in central and southern Italy confirmed the wide coenological amplitude of L. nobilis with respect to other laurophyllic species. Antica Lavinium has an overall good preservation of laurel forest formations, but also of mixed formation with $C$. australis. In the area, historical, cultural, and natural characteristics mutually contributed to the development of human civilizations and plant communities, highlighting their deep linkage.
\end{abstract}

Keywords: aeneas route; archaeological landscape; European hackberry; laurel; Mediterranean vegetation; plant history; relictuality

\section{Introduction}

The Mediterranean basin is one of the world's biodiversity hotspots [1]. Due to its richness in natural resources and geographical diversity, it is a mosaic of natural and cultural landscapes, where human civilizations and nature have coexisted for thousands of years [2]. In particular, the Italian territory homed Etruscans, Greeks, Latins, and several other indigenous or allochthonous populations, until the domination of the Roman civilization. Archaeobotanical studies identified several traces of human activities that influenced the landscape composition of central Italy (e.g., researchers have discovered pollen of arable crops dated about 5500 BCE [3]). The core area of the Roman civilization was in the Latium region and here the link between man and nature is still particularly evident, as for instance in the grasslands of the "Campagna romana" but also in the forest communities [4]. In this region, the Roman policy also caused major changes in the local vegetation, through ordered agricultural planning of the territory (centuria), and dismantling of some forests and wetlands, but also protecting others as sacred groves [5]. The human impact on the landscape of this area has been perpetuated over time, inducing changes in plant cover and floristic composition, spreading plants of economic and agricultural interest $[3,4,6]$, and sometimes creating relevant cultural landscapes [7]. Forests were a significant resource for the ancient Romans and the foundation and development of cities were closely related to their abundance in the territory [8]. Although the geographical expansion of the Roman Empire-which sparked a population increase, large-scale agriculture, and economic development-significantly increased deforestation and landscape modification, other 
conservative management practices and timber harvest systems, such as coppice, promoted forests' conservation [8].

Today, relict habitats, particularly in the Mediterranean basin, are of particular interest among the different habitats subjected to conservation programs and law protection. In fact, refugia are unanimously considered sites of special importance for biodiversity conservation [9], as they provide suitable habitats that limit species extinction and can favor taxa differentiation and speciation [10]. The Mediterranean basin constitutes a global refugium for relict plant taxa itself [10] and here few relict native laurophyllic forests survived, such as those located in the central and southern regions of Italy [11,12].

Here, both Laurel (Laurus nobilis L.) and the European hackberry (Celtis australis L.) show great importance for their relictuality and high historical and cultural relevance [13,14]. The presence of both species together is often fragmented, and the phytosociological classification of communities with both species has been differently interpreted. In fact, for Sardinia, a new association of small laurophyllic forest, which has L. nobilis and C. australis as its typical species, together with Ficus carica var. caprificus, has been described by Bacchetta et al. [15]. On the contrary, Gianguzzi et al. [16] and Marino et al. [17] have found in Sicily residual L. nobilis communities with a limited presence of C. australis, where the latter was not considered characteristic of the newly described association.

Within overall monitoring of the sites of natural interest in the Latium region, a Site of Community Importance (SCI, priority habitat 5230*) was identified within the Borghese Estate of Pratica di Mare [18]. Proposed to the European Union pursuant to the Habitat Directive 92/43/EEC, this site has been officially included in the Natura 2000 Network as a Special Area of Conservation (SAC) under the name "Antica Lavinium-Pratica di Mare". This area is also relevant from a historical point of view as Virgil, in his Aeneid, described Lavinium as the city founded by Aeneas and the Trojan exiles. The place has had for centuries a great symbolic value, because of this hero, the mythological founder of the gens Julia, and the first among the Founding Fathers (Patres) of Rome.

In the analysis of relict laurophyllic forests, communities with L. nobilis and C. australis have a significant role from a natural point of view, as well as for their historical and cultural values. Linking natural values with the material and immaterial heritage is a relevant issue for the site of Antica Lavinium-Pratica di Mare, such as for many other archaeological sites $[19,20]$. Such linkage is also highly underlined in the UNESCO-MAB (Man and Biosphere, https: / / en.unesco.org/mab, last accessed 26 November 2021) program, which aims to establish a scientific basis for enhancing the relationship between people (Man in the sense of humans) and their environments.

This paper is aimed at investigating the ecological and phytosociological framework of the relict laurophyllic communities of the site, as well as their cultural and historical values, within the valorization of ancient Mediterranean historical and cultural routes. Our work is also aimed at analyzing the conditions and factors that allowed their survival. These data will contribute to improving the management plan of the site, combining both naturalistic and cultural values.

\section{Materials and Methods}

\subsection{Study Site}

The Antica Lavinium site is located on a vast plateau in the Latium region, about $28 \mathrm{~km}$ south of Rome and about $4 \mathrm{~km}$ from the current coastline, which was closer to the site during the Roman times [21]. The site is bordered by steep slopes on the west and north sides, which have been carved by the river Fosso di Pratica di Mare, identified by some experts with the ancient Numicus river [21]. The study site coincides with the SAC Antica Lavinium area, which covers 48 hectares within the Borghese family's Estate of Pratica di Mare, and its borders overlap the property boundaries on the north side (bordering the Fosso di Pratica di Mare) and includes woodlands, an artificial lake, a small village, and a natural lake on the east side of the village. The surrounding area hosts the archaeological 
remains of Aeneas' heròon, the "Thirteen Ares" Sanctuary, and the Civic Archaeological Museum of Lavinium (Figure 1).

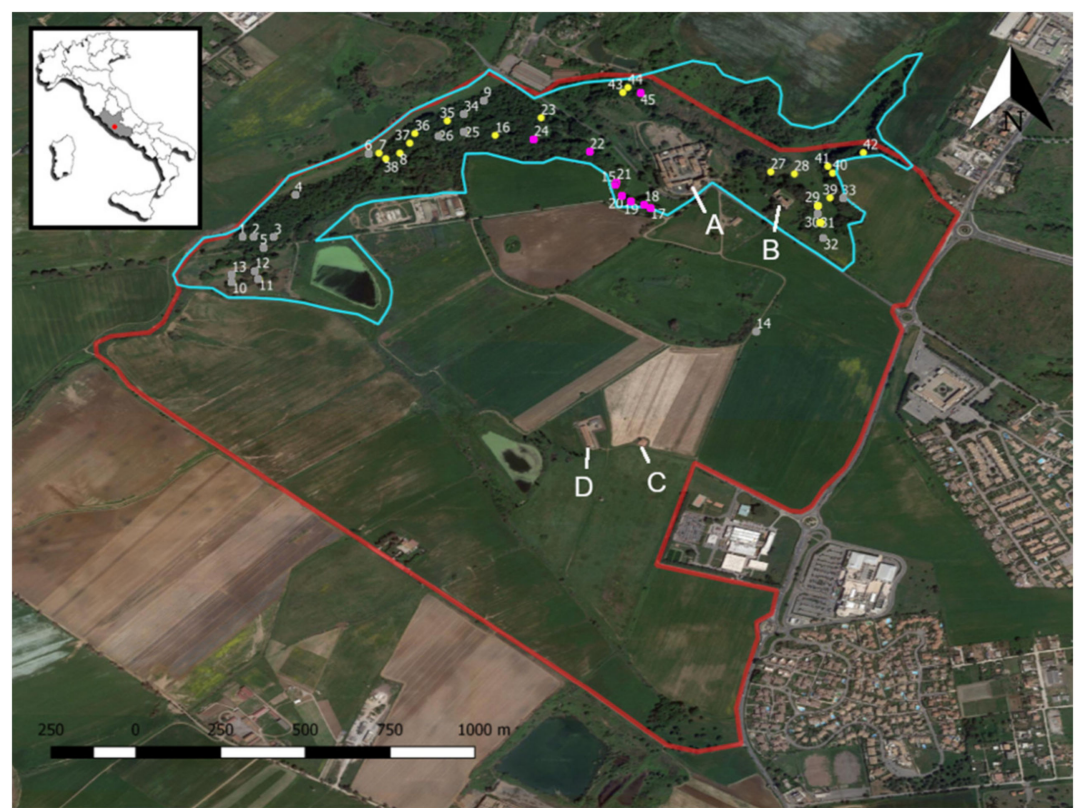

Figure 1. Map, from Google Earth, of the borders of the Estate (the red line) and of the study site (the SAC "Antica Lavinium", blue line); the numbered vegetation plots (yellow dots: samplings with pure L. nobilis formations; pink dots: samplings with mixed forests of L. nobilis and C. australis; grey dots: other samplings), and the buildings: the Pratica di Mare castle (A); the Civic Archaeological Museum of Lavinium (B); Aeneas' heròon (C); and the "Thirteen Ares" Sanctuary (D).

\subsubsection{Bioclimatic and Geological Features}

According to the Phytoclimatic Map of Italy [22], the site is characterized by a Mediterranean meso-temperate macroclimate, with a weakly transitional Mediterranean oceanic bioclimate, typical of the alluvial plains of the middle and upper Tyrrhenian Sea, which are affected by the more temperate climate of the innermost areas of the Alban Hills and the Monti Sabatini region. The average yearly rainfall is $794.8 \mathrm{~mm}$, with $17.4^{\circ} \mathrm{C}$ of average yearly temperature (meteorological data recorded from 2012 to 2017 by the "Pomezia sud" gauging station, from the hydrographic archive of the Latium region, http:/ / www.idrografico.regione.lazio.it/annali/index.htm, last accessed 26 November 2021). Rain mainly occurs during the autumn and winter seasons and is rarely above $100 \mathrm{~mm}$ per month, whilst summer is characterized by a dry period with rains below $50 \mathrm{~mm}$ per month and average temperatures above $20^{\circ} \mathrm{C}$.

The geology of the area is characterized by the presence of two formations of different origins. On the seaside, there are Eolic deposits, dating back to the Pleistocene-Holocene period (2.58 million-10,000 years ago) forming an unconsolidated lithological unit. On the inner side, there are volcanic deposits of foidites and tephrites (lavas, pyroclastites and ignimbrites), dating back to the Quaternary (1.65 million-10,000 years ago) (Computerized regional geological map, https:/ / geoportale.regione.lazio.it/layers/geonode: carta_geologica_wgs84, last accessed 26 November 2021). Similarly, the site is located between two different pedological regions, as it overlaps the region of the Tyrrhenian coastal plains of central Italy and the region of the volcanic hill areas of central and southern Italy (geological and pedological data from the Geoportale Nazionale, http: //www.pcn.minambiente.it/mattm/, last accessed 26 November 2021).

\subsubsection{Historical Background}

The historical origin of the Antica Lavinium settlement dates back to the first phase of the Iron age, as testified by the archaeological findings of the acropolis, the several 
tombs nearby, and the "Thirteen Ares" sanctuary [21]. The site then expanded and evolved across the centuries. Information about the settlement during the Middle Age is poor and unclear, although cartographic data show that in the first decades of the XV Century, the site was a hunting reserve of the Capranica family, and from the XVI Century, the modern name of Pratica was in use instead of Patrica ("father"). Since then, the site endured a succession of renovations, and the latest restoration and valorization projects started in 2008 (https: / / www.dimorestoricheitaliane.it/en/dimora/castello-borghese-pratica/, last accessed 26 November 2021). Besides historical documentation and archaeological findings, the origins of Antica Lavinium are enveloped in the mists of mythology. In fact, this site is reported by Virgil to be the landing site of Aenea, a key figure for the Roman culture. The lucus Sol Indiges or locus Solis Indigetis sanctuary, together with the Aeneas' heròn and the "Thirteen Ares" Sanctuary, are the archaeological remains that best represent the sacredness of this territory for the Romans [21,23].

\subsection{Data Analysis}

\subsubsection{Vegetation Data and Comparative Evaluations}

We performed vegetation surveys between April and June 2018 and in September 2018 in all the forested areas of the Borghese Estate within the protected SAC area (Figure 1), using the phytosociological approach of the Zurich-Montpellier School [24]. We carried out 45 forest surveys in the forest community within the Antica Lavinium SAC border. We identified the collected floristic samples using the Flora d'Italia [25], and we updated the nomenclature according to The Plant List site (http:/ / www.theplantlist.org/, last accessed 12 September 2021) and according to the checklist of the vascular native flora of Italy [26]. We processed the phytosociological data in an Excel spreadsheet, only including species found in at least two surveys, to avoid interferences of sporadic species. For each species, we visually estimated the cover percentage using the Braun-Blanquet's cover-abundance scale (. $=$ absent; $+=$ few individuals; $1=<5 \%$, numerous individuals; $2=5-25 \%$; $3=25-50 \%$; $4=50-75 \% ; 5=>75 \%$ ) [27]. We performed a Cluster Analysis with the Paired Group algorithm and the Bray-Curtis similarity measure, and a PCA (Principal Component Analysis) using the Past software (https:/ / palaeo-electronica.org/2001_1/past/issue1_01. htm, last accessed 12 September 2021).

Finally, we compared our relictual coenosis with pure L. nobilis formations [28-30], found in close Tyrrhenian areas (Tuscany and Latium) and mixed forests of L. nobilis and C. australis $[15,16,31]$ located in Sicily and Sardinia. To perform the comparative analysis, we firstly analyzed the patterns in species richness between datasets regarding the number of plant species in relation to the two different coenosis compositions (pure laurel formations and laurel-hackberry mixed formations), using Mann-Whitney $U$ test on Past software. Then, we used the Turboveg software package [32] for the storage of vegetation data, and subsequently, we exported our data to the Juice software [33] for an analysis of similarities and dissimilarities among samples using the ordination non-metric multidimensional scaling (NMDS).

\subsubsection{Historical and Cultural Species, Vegetation Data, and Site Management}

We examined the cultural and historical framework of the relictual species (L. nobilis and C. australis) and forests through an analysis of historical documentation and literature. We started with an analysis of works of ancient authors, i.e., Julius Obsequens, Valerius Maximus, Cato, Servius, Dionysius of Halicarnassus, Pliny the Elder, Ovid, and Virgil, sometimes using original textbooks, and sometimes their commentary notes and citations [34-36].

As a source of historical data and cultural value of the plants, we also considered the study on plant iconography in Greek [35,37] and Roman cultures [38-43] and on their rediscovery during the Renaissance [44]. We also considered the textbooks by Matthioli [45] on medicinal plants referred to Dioscorides and the further contributions by Durante [46]. Historical maps of the coastal areas, such as Topografia Geometrica dell'Agro Romano by 
Giovanni Battista Cingolani, 1689 and further examples were also considered to evaluate the antiquity of the forest.

Furthermore, we consulted the archives of the Superintendence of Cultural Heritage and we collected information on the historical management carried out by the Borghese family, owner of the Estate since the XVII Century. We also considered the results of the archaeological research conducted in the Antica Lavinium [21,23,47,48], which often provide interesting information on the natural landscape. Finally, we consulted ethnobotanical recent literature to determine the uses of L. nobilis and C. australis $[49,50]$.

\subsubsection{Assessment of the Conservation of Natural and Cultural Values}

We firstly evaluated the conservation status of the analyzed forests through literature data, and subsequently, the six yearly SAC reports required by the Habitat Directive and then through an on-field analysis. We checked the status of such habitats in the International Directives (Habitats Directive 92/43/EEC and CITES - Convention on International Trade in Endangered Species of Wild Fauna and Flora), National and Regional Red Lists [51-55], the Latium Regional Law List of Rare or Vulnerable species (L.R. 19 September 1974, n. 61), and other lists for endemic and rare species [56]. We consulted pertinent and recent literature to obtain information on the vegetation communities' distribution [57-60].

After the field analysis, for each detected species we also considered conservation interest, rarity, vulnerability, endemism, and linkage with specific habitats $[19,20]$. Furthermore, we considered critical situations for biodiversity conservation, assessing the presence and distribution of exotic species and their invasiveness [61]. The assessment of the conservation of cultural values of L. nobilis and C. australis was performed using the literature cited in Section 2.2.2 and listing their frequency in the Antica Lavinium site $[19,20]$. Finally, to assess the importance and cultural values of the identified plants, we considered the protection laws and the management strategies by the Authorities and by the Estate.

\section{Results}

\subsection{Vegetation Analysis and Comparative Evaluations}

Among the 45 forest surveys, 25 vegetation samplings were characterized by the target species (L. nobilis and C. australis; Figure 2, Table 1). Species of the genus Quercus resulted as key elements of the vegetation composition of the site (i.e., Q. suber and $Q$. ilex in the thermophilic sector, and Q. cerris and Q. robur in the more humid and cooler sector).

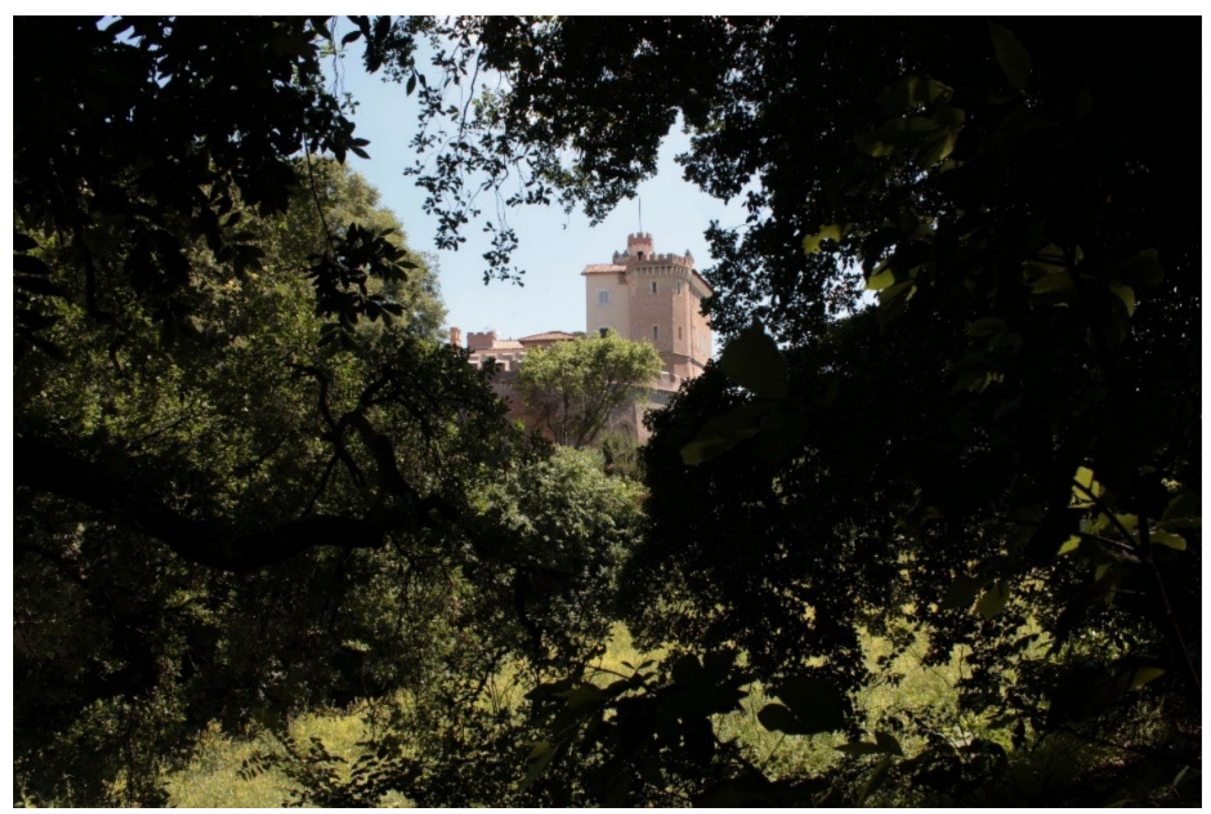

Figure 2. The castle of Pratica di Mare as seen from the forest sector of the Estate (A) with an example of one of the tallest hackberry trees. 
Table 1. Relevés of L. nobilis and C. australis woodlands in the Antica Lavinium (Pratica di Mare) site. Cover percentages are reported according to the Braun-Blanquet scale.

\begin{tabular}{|c|c|c|c|c|c|c|c|c|c|c|c|c|c|c|c|c|c|c|c|c|c|c|c|c|c|}
\hline \multicolumn{26}{|c|}{ Relevés of Laurus nobilis and Celtis australis in the Antica Lavinium (Pratica di Mare) Site } \\
\hline $\mathbf{N}^{\circ}$ of relevé & 1 & 2 & 3 & 4 & 5 & 6 & 7 & 8 & 9 & 10 & 11 & 12 & 13 & 14 & 15 & 16 & 17 & 18 & 19 & 20 & 21 & 22 & 232 & 24 & 2 \\
\hline Altitude (m) & 83 & 93 & 80 & 65 & 84 & 84 & 84 & 70 & 73 & 67 & 65 & 108 & 63 & 61 & 83 & 83 & 83 & 49 & 81 & 80 & 76 & 50 & 50 & 61 & 6 \\
\hline Inclinatic & 40 & 30 & 30 & 15 & 36 & 35 & 35 & 35 & 35 & 40 & 15 & 40 & 40 & 60 & 15 & 15 & 15 & 15 & 45 & 40 & 40 & 403 & 35 & & \\
\hline Aspect & NW & NW & $\mathrm{NE}$ & NE & $\mathrm{N}$ & $\mathrm{N}$ & $\mathrm{NE}$ & $\mathrm{NE}$ & $\mathrm{NE}$ & $\mathrm{NE}$ & NE & $\mathrm{NE}$ & $\mathrm{N}$ & $\mathrm{NE}$ & NW & NW & NW & NW & $\mathrm{W}$ & NW & NW & $\mathrm{N}$ & & $\mathrm{NE}$ & $\mathrm{EN}$ \\
\hline Veg. heigl & 15 & 20 & 15 & 15 & 15 & 18 & 18 & 18 & 15 & 18 & 15 & 15 & 13 & 18 & 15 & 15 & 16 & 10 & 18 & 18 & 18 & 10 & 161 & 18 & 18 \\
\hline Cover & 80 & 80 & 90 & 90 & 75 & 75 & 75 & 75 & 75 & 60 & 75 & 60 & 80 & 70 & 80 & 80 & 70 & 70 & 70 & 80 & 60 & 607 & 707 & 75 & 75 \\
\hline Relevé area $\left(\mathrm{m}^{2}\right)$ & 100 & 100 & 600 & 400 & 100 & 400 & 200 & 200 & 100 & 100 & 400 & 100 & 200 & 200 & 200 & 200 & 75 & 75 & & 0400 & 100 & 400 & 4004 & 400 & 04 \\
\hline Total $n^{\circ}$ of species & 17 & 18 & 30 & 24 & 8 & 22 & 15 & 10 & 7 & 7 & 18 & 17 & 14 & 7 & 14 & 11 & 9 & 7 & 10 & 14 & 7 & 15 & 16 & & 6 \\
\hline & 4 & 3 & 2 & 3 & 2 & 3 & 2 & 3 & 2 & 3 & 3 & 4 & 4 & 4 & 2 & 2 & 3 & 4 & 3 & 3 & 3 & 2 & 2 & 2 & \\
\hline \multicolumn{26}{|c|}{ Diff. Ass. Celtido australis-Lauretum nobilis Bacchetta, Farris, Fenu, Filigheddu, Mattana and Mulè 2007} \\
\hline Celtis australis & . & . & 4 & . & + & 2 & 3 & 4 & 3 & 4 & . & + & . & . & . & . & & . & . & . & . & . & 1 & & \\
\hline Quercus ilex & & & + & + & & & . & & 2 & . & + & & 4 & 3 & & & & . & 3 & 3 & 3 & & & & 3 \\
\hline \multicolumn{26}{|c|}{ Diff. Ass. Acantho mollis-Lauretum nobilis Gianguzzi, D'Amico and Romano 2010} \\
\hline Hedera helix & + & 3 & + & 1 & 3 & 2 & 1 & + & & . & 4 & + & 1 & 2 & 2 & 3 & 3 & 1 & & . & + & 3 & + & + & \\
\hline Acanthus mollis & & & & 1 & . & & & . & & . & . & 3 & . & . & . & . & . & . & . & . & . & . & . & . & \\
\hline \multicolumn{26}{|c|}{ All. Fraxino orni-Quercion ilicis Biondi, Casavecchia and Gigante 2003} \\
\hline Dioscorea communis & + & 1 & 3 & 1 & + & 2 & 1 & 2 & 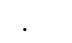 & 1 & r & + & & $\cdot$ & & + & & + & . & + & . & + & & & \\
\hline & . & . & + & . & . & . & . & . & . & + & + & & & . & . & & & . & . & . & . & . & . & . & . \\
\hline & + & 1 & + & + & . & . & . & . & . & + & + & 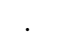 & . & . & + & . & & + & . & . & . & 1 & + & . & . \\
\hline & . & . & + & 2 & . & . & . & . & 1 & . & . & 1 & + & 1 & 3 & . & & 2 & . & 2 & 1 & . & . & . & . \\
\hline Phillyrea latifolia & & + & + & + & . & & 1 & . & + & . & + & 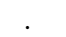 & 2 & - & . & . & & + & + & . & 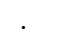 & & & & \\
\hline \multicolumn{26}{|c|}{ Cl. Quercetea ilicis Br.-Bl. in Br.-Bl., Roussine and Nègre 1952} \\
\hline Asple & . & & + & + & . & + & . & & & & & + & 1 & & & & & & + & + & + & . & . & . & \\
\hline & 2 & 2 & 2 & 4 & + & + & + & 2 & 3 & 2 & 3 & 2 & & . & 2 & 2 & 2 & 2 & 1 & 1 & + & + & + & & \\
\hline & + & + & + & 2 & . & . & + & 1 & . & + & . & . & ? & . & 1 & 1 & . & . & 3 & 1 & . & 2 & + & 2 & 2 \\
\hline & . & . & + & + & + & + & . & . & . & . & . & . & + & . & . & . & . & . & + & 1 & . & + & + & 1 & \\
\hline & . & 1 & + & . & . & . & . & . & . & . & & 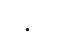 & . & & & . & . & . & . & . & . & . & 2 & & \\
\hline & + & . & + & . & 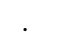 & + & + & . & . & . & + & . & + & 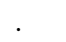 & . & & + & . & 2 & + & . & . & + & & \\
\hline & . & . & 1 & . & . & + & 1 & . & . & . & te & + & . & . & & . & . & . & + & . & . & 2 & + & 2 & 3 \\
\hline Viburnum & & & + & + & & 1 & & & & . & + & + & . & 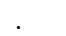 & 1 & & & . & . & + & . & 2 & 3 & & \\
\hline \multicolumn{26}{|c|}{ Cl. Querco roboris-Fagetea sylvaticae Br.-Bl. and Vlieger in Vlieger 1937} \\
\hline & 1 & 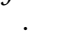 & + & + & 3 & 2 & . & + & + & . & . & $\cdot$ & . & + & . & 1 & & . & . & + & . & . & . & + & \\
\hline & . & 1 & + & + & . & 3 & . & 2 & . & . & + & + & . & . & 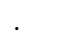 & & & . & . & . & . & . & . & . & . \\
\hline & . & . & . & . & . & . & . & . & . & . & . & . & . & . & + & 3 & 3 & . & . & . & . & . & . & . & . \\
\hline & 2 & 1 & . & . & . & . & . & . & . & . & 2 & + & . & + & & 1 & + & . & . & 1 & . & . & . & . & \\
\hline & . & . & . & + & 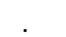 & . & . & . & 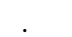 & 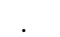 & & & 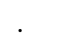 & 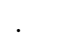 & & 1 & 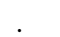 & . & . & 1 & . & . & . & . & \\
\hline & . & 4 & + & 3 & . & 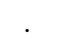 & 1 & & 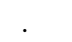 & $\cdot$ & 4 & + & 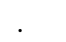 & 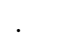 & 3 & . & & . & . & . & . & 4 & 3 & & \\
\hline & . & . & . & 2 & . & . & . & 1 & . & . & + & . & . & . & . & . & & . & . & . & . & . &. & . & . \\
\hline & . & . & . & . & . & + & + & . & . & . & . & . & . & . & . & . & 4 & . & . & . & . & . & . & . & . \\
\hline & & & + & + & . & 1 & . & . & 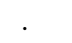 & . & . & . & . & . & . & . & & . & . & . & . & 1 & & & \\
\hline Ficaria verna & 1 & 2 & . & . & & . & $\cdot$ & & & & $\cdot$ & & & & & & & . & & . & 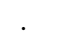 & . & & & . \\
\hline \multicolumn{26}{|c|}{ Cl. Salici purpureae-Populetea nigrae Rivas-Martínez, T.E. Diaz, Fernandez-Gonzalez, Izco, Loidi, Lousa and Penas 2002} \\
\hline $\begin{array}{l}\text { Arum italicum } \\
\text { Cl. Rhamno cathartic }\end{array}$ & \multirow{2}{*}{\multicolumn{19}{|c|}{ ae-Prunetea spinosae Tüxen 1952}} & . & . & . & . & . & . \\
\hline & e-Pru & netea & $\begin{array}{c}\text { Spinc } \\
+\end{array}$ & $\begin{array}{c}\text { sae } \mathrm{T} \\
+\end{array}$ & tuxen & & & & & & & & & & & & & & & & & & & & \\
\hline & $\cdot$ & . & + & $T$ & $\cdot$ & 1 & 1 & . & & & . & . & . & $\cdot$ & & . & + & . & 1 & . & + & . & 1 & & \\
\hline & . & $\cdot$ & + & 1 & . & 1 & . & . & $\cdot$ & $\cdot$ & + & & & $\cdot$ & 1 & . & & . & . & + & . & + & 1 & 1 & \\
\hline & . & . & + & . & + & + & . & . & . & . & . & + & 1 & + & 1 & . & & . & . & . & . & 1 & + & 4 & 1 \\
\hline $\begin{array}{l}\text { Fraxinus angustifolia } \\
\text { subsp. Oxycarpa }\end{array}$ & 2 & 2 & 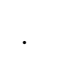 & . & . & . & . & . & . & . & 2 & . & . & . & . & 1 & & . & . & & . & . & & & \\
\hline Nitrophilic compone & & & & & & & & & & & & & & & & & & & & & & & & & \\
\hline & 1 & . & 1 & . & $\cdot$ & 2 & 1 & . & . & . & . & . & + & . & . & . & & . & . & . & . & . & & & \\
\hline & + & + & + & + & . & + & 1 & & & 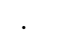 & 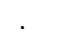 & + & & $\cdot$ & . & . & & . & . & & . & + & & & \\
\hline & + & + & + & . & . & + & + & $\cdot$ & 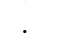 & . & . & . & 1 & . & . & . & & . & . & . & . & . & & & \\
\hline & . & & + & + & . & + & . & + & & . & . & . & . & . & . & . & & . & . & . & . & . & & & \\
\hline Allium & 1 & 1 & . & . & . & . & . & . & 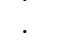 & . & . & + & . & . & . & . & . & . & . & . & . & . & . & . & \\
\hline $\begin{array}{l}\text { Ranun } \\
\text { lanugir }\end{array}$ & 1 & 1 & & + & . & & . & . & . & . & . & 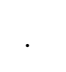 & . & . & . & . & 1 &. & . & . & . & . & . & & \\
\hline
\end{tabular}


The PCA analysis highlighted how the two detected types of formations present substantial differences (Figure 3). The L. nobilis formations (black dots) have a wide distribution in the graph, so the differential elements are represented by Quercus ilex, Q. cerris and Acer campestre. Comparatively, in the mixed formations (C. australis and L. nobilis; pink dots) C. australis appeared to be the principal component of the coenosis. In the comparative analysis (Table 2) between our coenosis and pure L. nobilis formations [28-30] or mixed forests with L. nobilis and C. australis $[15,16,31]$, the major differences seemed to be related to the higher presence (in number and cover) of mesophilous species (Cl. Querco-Fagetea) in the first group of surveys (Latium and Tuscany), while the second group of surveys showed a more typical Mediterranean composition (Cl. Quercetea ilicis). Moreover, from the comparative analysis of the floristic richness, no statistically significant differences between the pure laurel and laurel-hackberry mixed formations emerged $(p=0.46$; Mann-Whitney $U$ test).

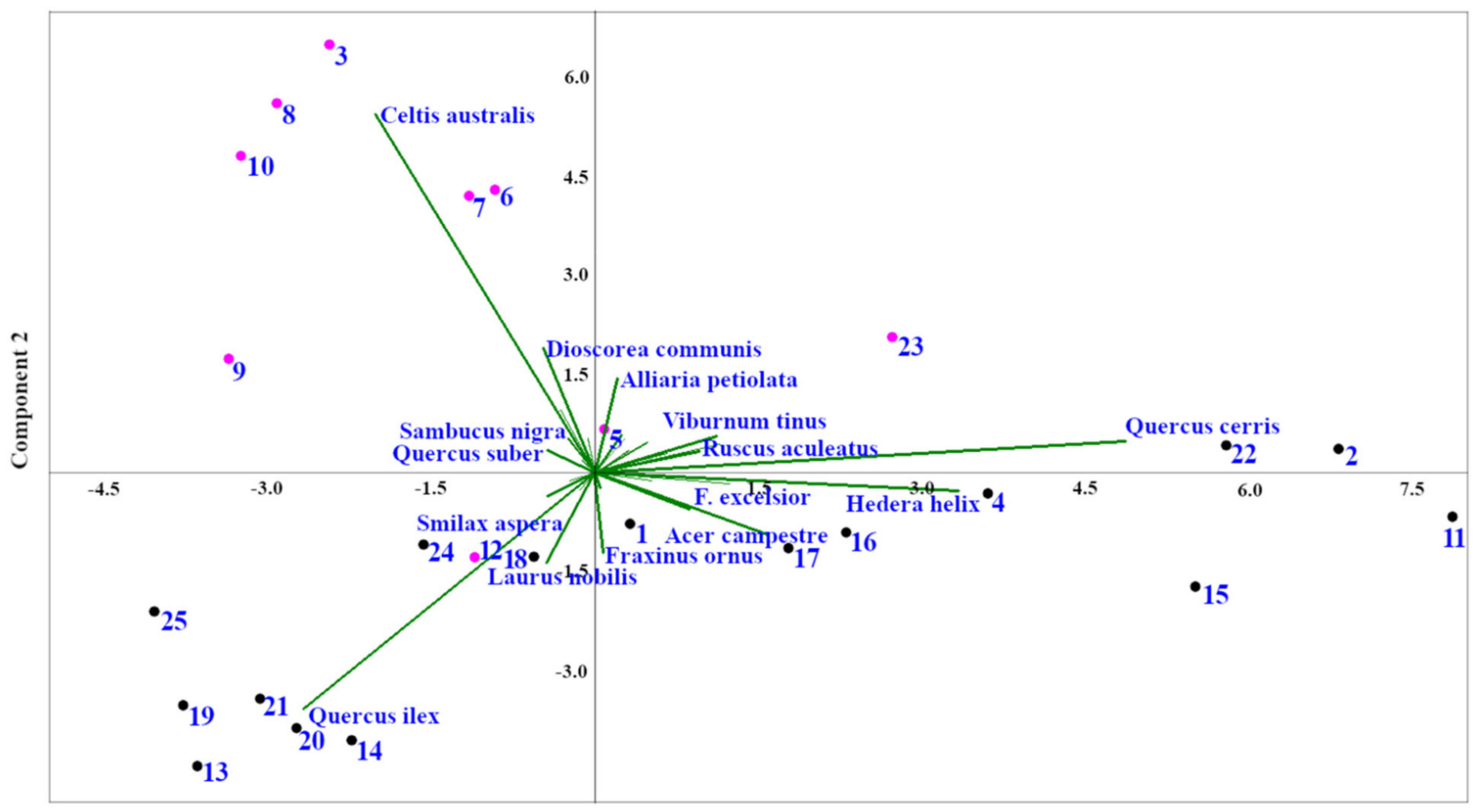

Component 1

Figure 3. Principal Component Analysis (PCA) of the laurel forest formations of the Antica Lavinium SAC. (Numbers corresponds to the relevès of Table 1). Sampling plots with co-dominance of L. nobilis and $C$. australis are represented by pink dots, while black dots represent plots where $C$. australis was not found and L. nobilis is associated with different forest species. Weighted presence of the species within the samplings is represented by green lines, for which some of the most ecologically significant species are reported. 
Table 2. Synoptic table of the comparison between pure L. nobilis coenoses and the mixed L. nobilis and C. australis coenoses.

\begin{tabular}{|c|c|c|c|c|c|c|c|}
\hline \multicolumn{8}{|c|}{ Synoptic Table } \\
\hline Placement & $\begin{array}{l}\text { Latium } \\
\text { (Antica } \\
\text { Lavium) }\end{array}$ & $\begin{array}{c}\text { Latium } \\
\text { (Coastal Zone) }\end{array}$ & $\begin{array}{l}\text { Latium (Ponte } \\
\text { Renaro) }\end{array}$ & $\begin{array}{c}\text { Tuscany } \\
\text { (Argentario) }\end{array}$ & $\begin{array}{c}\text { Sardinia } \\
\text { (North } \\
\text { Western Zone) }\end{array}$ & $\begin{array}{c}\text { Sicily } \\
\text { (Hyblaean } \\
\text { Mountains) }\end{array}$ & $\begin{array}{c}\text { Sicily } \\
\text { (Mountain } \\
\text { Areas, } \\
\text { Scattered Zones) }\end{array}$ \\
\hline References & Relevès 2018 & $\begin{array}{l}\text { Lucchese and } \\
\text { Pignatti [28] }\end{array}$ & $\begin{array}{c}\text { Pignatti et al. } \\
{[30]}\end{array}$ & $\begin{array}{c}\text { Arrigoni and } \\
\text { Di Tommaso } \\
\text { [29] }\end{array}$ & $\begin{array}{c}\text { Bacchetta et al. } \\
{[15]}\end{array}$ & $\begin{array}{l}\text { Brullo et al. } \\
\text { [31] }\end{array}$ & $\begin{array}{c}\text { Gianguzzi et al. } \\
\text { [16] }\end{array}$ \\
\hline $\mathrm{N}^{\circ}$ of relevé & 25 & 11 & 7 & 3 & 15 & 10 & 18 \\
\hline \multicolumn{8}{|l|}{ Car. Ass. } \\
\hline \multicolumn{8}{|c|}{ Diff. Ass. Celtido australis-Lauretum nobilis Bacchetta, Farris, Fenu, Filigheddu, Mattana and Mulè 2007} \\
\hline Celtis australis & II & & & & III & III & I \\
\hline Ficus carica & & & & & III & II & III \\
\hline Quercus ilex & III & IV & III & 3 & III & I & I \\
\hline \multicolumn{8}{|c|}{ Diff. Ass. Acantho mollis-Lauretum nobilis Gianguzzi, D'Amico and Romano 2010} \\
\hline Hedera helix & IV & $\mathrm{V}$ & $\mathrm{V}$ & 3 & $\mathrm{~V}$ & $\mathrm{~V}$ & $\mathrm{~V}$ \\
\hline $\begin{array}{l}\text { Pistacia } \\
\text { terebinthus }\end{array}$ & & & & & & II & IV \\
\hline Acanthus mollis & I & & & & I & $\mathrm{V}$ & $\mathrm{V}$ \\
\hline $\begin{array}{l}\text { Orobanche } \\
\text { hederae }\end{array}$ & & & & & & I & II \\
\hline \multicolumn{8}{|c|}{ Diff. Ass. Lauro Carpinetum betuli Lucchese and Pignatti 1990} \\
\hline $\begin{array}{l}\text { Carpinus } \\
\text { betulus }\end{array}$ & & IV & V & & & & \\
\hline $\begin{array}{l}\text { Polystichum } \\
\text { setiferum }\end{array}$ & & I & & & & & \\
\hline $\begin{array}{l}\text { Phyllitis } \\
\text { scolopendrium }\end{array}$ & & I & & & & & \\
\hline \multicolumn{8}{|c|}{ All. Quercion frainetto cfr. Lucchese and Pignatti 1990} \\
\hline $\begin{array}{l}\text { Anemone } \\
\text { apennina }\end{array}$ & & II & II & & & & \\
\hline $\begin{array}{l}\text { Lathyrus } \\
\text { venetus }\end{array}$ & & II & & & & & \\
\hline $\begin{array}{l}\text { Ranunculus } \\
\text { lanuginosus }\end{array}$ & & II & & & & & \\
\hline $\begin{array}{l}\text { Sorbus } \\
\text { domestica }\end{array}$ & & I & & & & & \\
\hline Lonicera etrusca & & I & & & & & \\
\hline $\begin{array}{l}\text { Buglossoides } \\
\text { purpurocaerulea }\end{array}$ & & I & & & & & \\
\hline $\begin{array}{l}\text { Mespilus } \\
\text { germanica }\end{array}$ & & I & & & & & \\
\hline $\begin{array}{l}\text { Pyrus } \\
\text { amygdaliformis }\end{array}$ & & I & & & & & \\
\hline $\begin{array}{l}\text { Oenanthe } \\
\text { pimpinelloides }\end{array}$ & & I & & & & & \\
\hline \multicolumn{8}{|c|}{ All. Fraxino orni-Quercion ilicis Biondi, Casavecchia and Gigante 2003} \\
\hline $\begin{array}{l}\text { Cyclamen } \\
\text { hederifolium }\end{array}$ & II & & & & & $\mathrm{V}$ & $\mathrm{V}$ \\
\hline $\begin{array}{l}\text { Dioscorea } \\
\text { communis }\end{array}$ & IV & IV & I & 3 & $\mathrm{~V}$ & I & IV \\
\hline Fraxinus ornus & II & III & II & 3 & & & II \\
\hline $\begin{array}{l}\text { Phillyrea } \\
\text { latifolia }\end{array}$ & II & & IV & 2 & III & & \\
\hline $\begin{array}{l}\text { Clematis } \\
\text { cirrhosa }\end{array}$ & & & & & II & II & \\
\hline $\begin{array}{l}\text { Cyclamen } \\
\text { repandum }\end{array}$ & I & III & & 1 & II & & \\
\hline
\end{tabular}


Table 2. Cont.

\begin{tabular}{|c|c|c|c|c|c|c|c|}
\hline \multicolumn{8}{|c|}{ Synoptic Table } \\
\hline Placement & $\begin{array}{l}\text { Latium } \\
\text { (Antica } \\
\text { Lavium) }\end{array}$ & $\begin{array}{c}\text { Latium } \\
\text { (Coastal Zone) }\end{array}$ & $\begin{array}{l}\text { Latium (Ponte } \\
\text { Renaro) }\end{array}$ & $\begin{array}{c}\text { Tuscany } \\
\text { (Argentario) }\end{array}$ & $\begin{array}{c}\text { Sardinia } \\
\text { (North } \\
\text { Western Zone) }\end{array}$ & $\begin{array}{c}\text { Sicily } \\
\text { (Hyblaean } \\
\text { Mountains) }\end{array}$ & $\begin{array}{c}\text { Sicily } \\
\text { (Mountain } \\
\text { Areas, } \\
\text { Scattered Zones) }\end{array}$ \\
\hline \multicolumn{8}{|c|}{ Cl. Quercetea ilicis Br.-B1. in Br.-Bl., Roussine and Nègre 1952} \\
\hline $\begin{array}{l}\text { Ruscus } \\
\text { aculeatus }\end{array}$ & V & V & V & 2 & IV & $\mathrm{V}$ & I \\
\hline Smilax aspera & IV & I & III & 2 & IV & III & IV \\
\hline $\begin{array}{l}\text { Asparagus } \\
\text { acutifolius }\end{array}$ & II & II & I & & IV & III & $\mathrm{V}$ \\
\hline $\begin{array}{l}\text { Allium } \\
\text { subhirsutum }\end{array}$ & & & & & & I & $\mathrm{V}$ \\
\hline $\begin{array}{l}\text { Arisarum } \\
\text { vulgare }\end{array}$ & & & III & & III & & $\mathrm{V}$ \\
\hline $\begin{array}{l}\text { Asplenium } \\
\text { onopteris }\end{array}$ & II & I & III & 1 & IV & I & I \\
\hline $\begin{array}{l}\text { Rhamnus } \\
\text { alaternus }\end{array}$ & I & & & & II & $\mathrm{V}$ & III \\
\hline $\begin{array}{l}\text { Rosa } \\
\text { sempervirens }\end{array}$ & & & II & 2 & II & IV & II \\
\hline $\begin{array}{l}\text { Rubia peregrina } \\
\text { Rubia peregrina }\end{array}$ & II & I & III & 1 & & IV & \\
\hline $\begin{array}{l}\text { subsp. } \\
\text { longifolia }\end{array}$ & & & & & & & $\mathrm{V}$ \\
\hline Arbutus unedo & & & & 3 & & & \\
\hline Carex distachya & & & I & & I & & II \\
\hline Erica arborea & & & & 3 & & & \\
\hline $\begin{array}{l}\text { Myrtus } \\
\text { communis }\end{array}$ & & & & 1 & & & \\
\hline Osyris alba & & & & & & III & III \\
\hline Quercus suber & II & & & & & & \\
\hline $\begin{array}{l}\text { Quercus } \\
\text { virgiliana }\end{array}$ & & & & & & & II \\
\hline $\begin{array}{l}\text { Rubia peregrina } \\
\text { subsp. } \\
\text { peregrina }\end{array}$ & & & & & II & & \\
\hline $\begin{array}{l}\text { Chamaerops } \\
\text { humilis }\end{array}$ & & & & & & & I \\
\hline Viburnum tinus & II & II & III & 2 & & & I \\
\hline \multicolumn{8}{|c|}{ Cl. Querco roboris-Fagetea sylvaticae Br.-Bl. and Vlieger in Vlieger 1937} \\
\hline $\begin{array}{l}\text { Brachypodium } \\
\text { sylvaticum }\end{array}$ & & III & 0 & & & IV & II \\
\hline Acer campestre & II & IV & III & & & & \\
\hline Alliaria petiolata & II & I & II & & & & \\
\hline Carex pendula & $\mathrm{I}$ & & & & & & I \\
\hline $\begin{array}{l}\text { Ostrya } \\
\text { carpinifolia }\end{array}$ & & I & & 2 & & & \\
\hline Clematis vitalba & & II & II & & II & II & \\
\hline Cornus mas & I & IV & II & & & & \\
\hline Ilex aquifolium & & & & 3 & & & \\
\hline Luzula forsteri & & I & II & & & & \\
\hline Melica uniflora & & IV & II & & & & \\
\hline Quercus cerris & II & IV & & & & & \\
\hline $\begin{array}{l}\text { Quercus } \\
\text { pubescens }\end{array}$ & I & I & & 3 & & & \\
\hline $\begin{array}{l}\text { Acer monspessu- } \\
\text { lanum }\end{array}$ & & I & & 2 & & & \\
\hline $\begin{array}{l}\text { Sorbus } \\
\text { torminalis }\end{array}$ & & I & & 2 & & & \\
\hline Quercus robur & III & & II & & & & \\
\hline Ajuga reptans & & I & III & & & & \\
\hline Carex sylvatica & & II & II & & & & \\
\hline Corylus avellana & & I & III & & & & \\
\hline
\end{tabular}


Table 2. Cont.

\begin{tabular}{|c|c|c|c|c|c|c|c|}
\hline \multicolumn{8}{|c|}{ Synoptic Table } \\
\hline Placement & $\begin{array}{l}\text { Latium } \\
\text { (Antica } \\
\text { Lavium) }\end{array}$ & $\begin{array}{c}\text { Latium } \\
\text { (Coastal Zone) }\end{array}$ & $\begin{array}{l}\text { Latium (Ponte } \\
\text { Renaro) }\end{array}$ & $\begin{array}{c}\text { Tuscany } \\
\text { (Argentario) }\end{array}$ & $\begin{array}{c}\text { Sardinia } \\
\text { (North } \\
\text { Western Zone) }\end{array}$ & $\begin{array}{c}\text { Sicily } \\
\text { (Hyblaean } \\
\text { Mountains) }\end{array}$ & $\begin{array}{c}\text { Sicily } \\
\text { (Mountain } \\
\text { Areas, } \\
\text { Scattered Zones) }\end{array}$ \\
\hline $\begin{array}{l}\text { Euphorbia } \\
\text { amygdaloides }\end{array}$ & & I & II & & & & \\
\hline Ficaria verna & & I & III & & & & \\
\hline $\begin{array}{l}\text { Ligustrum } \\
\text { vulgare }\end{array}$ & & II & & & & & \\
\hline Prunus spinosa & & II & & & & & \\
\hline & & II & & & & & \\
\hline $\begin{array}{l}\text { sanguinea } \\
\text { Emerus major }\end{array}$ & & II & & & & & \\
\hline \multicolumn{8}{|c|}{ Cl. Salici purpureae-Populetea nigrae Rivas-Martínez, T.E. Diaz, Fernandez-Gonzalez, Izco, Loidi, Lousa and Penas 2002} \\
\hline Populus nigra & & & & & & & II \\
\hline Populus tremula & & & & 2 & & & \\
\hline Vitis sylvestris & & & & 1 & & & \\
\hline Arum italicum & I & III & & & II & IV & IV \\
\hline Vitis sylvestris & & & & & II & & \\
\hline \multicolumn{8}{|c|}{ Cl. Rhamno catharticae-Prunetea spinosae Tüxen 1952} \\
\hline $\begin{array}{l}\text { Crataegus } \\
\text { monogyna }\end{array}$ & II & II & III & 3 & I & I & \\
\hline $\begin{array}{l}\text { Rubus } \\
\text { ulmifolius }\end{array}$ & II & & I & & IV & V & \\
\hline $\begin{array}{l}\text { Ulmus minor } \\
\text { Asplenium }\end{array}$ & III & II & II & & & III & II \\
\hline $\begin{array}{l}\text { trichomanes } \\
\text { subsp. } \\
\text { quadrivalens }\end{array}$ & & & & & II & & \\
\hline $\begin{array}{l}\text { Euonymus } \\
\text { europaeus }\end{array}$ & II & III & I & & & & \\
\hline $\begin{array}{l}\text { Euphorbia } \\
\text { characias }\end{array}$ & & & & & & & III \\
\hline Rhamus oleoides & & & & & & & I \\
\hline Sambucus nigra & II & & & & I & I & \\
\hline \multicolumn{8}{|c|}{ Nitrophilic component } \\
\hline $\begin{array}{l}\text { Allium } \\
\text { triquetrum }\end{array}$ & I & I & & & II & & \\
\hline $\begin{array}{l}\text { Dryopteris } \\
\text { pallida }\end{array}$ & & & & & & II & \\
\hline Galium aparine & II & & & & I & & \\
\hline $\begin{array}{l}\text { Parietaria } \\
\text { judaica }\end{array}$ & & & & & & II & \\
\hline $\begin{array}{l}\text { Polypodium } \\
\text { cambricum }\end{array}$ & & & III & & & & \\
\hline $\begin{array}{l}\text { Polypodium } \\
\text { cambricum } \\
\text { subsp. } \\
\text { serrulatum }\end{array}$ & & & & & III & & \\
\hline $\begin{array}{l}\text { Ranunculus } \\
\text { lanuginosus } \\
\text { Robinia } \\
\text { pseudoacacia }\end{array}$ & I & & II & & & & \\
\hline Stellaria media & II & & & & & & \\
\hline Urtica dioica & $\mathrm{I}$ & & & & I & & \\
\hline Urtica rupestris & & & & & & III & \\
\hline
\end{tabular}

The NMDS analysis (Figure 4) highlighted the wide spatial distribution of all the Antica Lavinium plots, indicating an evident ecological gradient in a relatively small area. At the same time, a clear separation between plots with pure laurel (full blue circles) and mixed laurel and hackberry formations (empty blue circles) was not evident for our communities, nor for others in the Italian territories. The NMDS graph showed a clear separation just in the case of the Sicilian surveys; moreover, a significant overlap can be observed between 
the forest laurel formation of Antica Lavinium, and the formations of pure laurel described by Lucchese and Pignatti [28], Arrigoni and Di Tommaso [29] and Pignatti et al. [30].

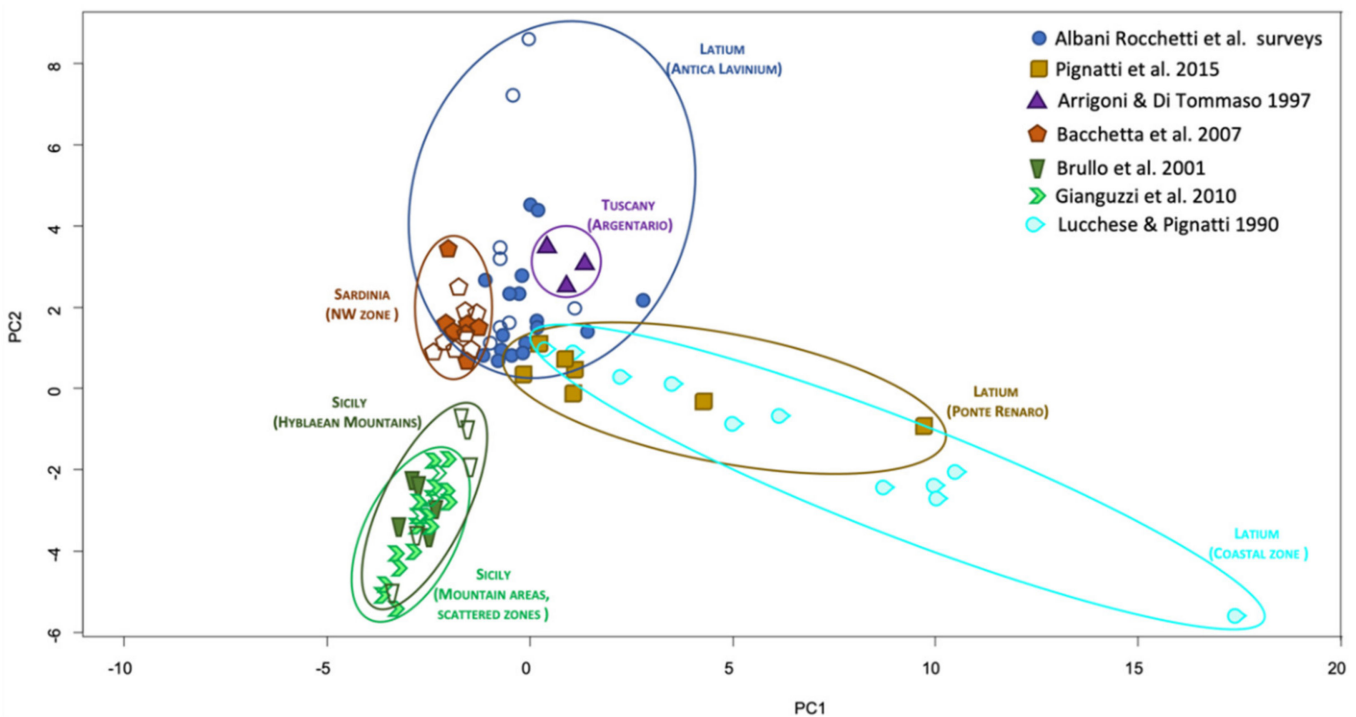

Figure 4. Non metric multidimensional scaling (NMDS). Comparative analysis of the laurel forest formations described in this study with those found in other sites of central Italy and islands (Sicily and Sardinia). (Full color icons $=$ L. nobilis pure formations; empty color icons $=$ L. nobilis and C. australis formations.)

\subsection{Historical and Cultural Analysis of Species and Forests and Their Management}

The analysis of historical documentation and literature confirmed, for both laurel and European hackberry, the high cultural value of the plants as widely reported in myths, celebrations and uses in Roman and Greek cultures. Laurel was the sacred plant of Apollo (the God of the Sun) [35]; Greeks used laurel as a symbol of victory or of highest status (e.g., in Pythian Games where a wreath of laurel was presented to winners). It was used as a symbol of the Roman imperial royalty by Augustus, who chose it as the emblem of the Roman emperors (Figure 5). During the Roman triumphs (the celebration and sanctification ceremonies of successful military commanders), commanders girded their heads with laurel leaves collected from the sacred grove of Livia's residence [44]. Ancient populations also made extensive use of leaves of laurel as a spice in food, decorations, and in medicine, and such uses are still preserved today, as well as its augural value $[62,63]$.

C. australis is cited in ancient Greek literature and it was also appreciated for its qualities by the Romans [64]. Pliny the Elder (Naturalis Historia) reports the existence of different varieties of hackberry ("lotus"), which were appreciated for their fruits (from which a very sweet wine was obtained), for its wood (used for creating flutes), and for its roots (from which knives, handles, and other small items were obtained). It was highly appreciated as an ornamental tree due to its dome-shaped crown [65].

When considering the Antica Lavinium site, in the past, the territory was widely forested, as described by Julius Obsequens ("silva Laurentina", IV sec BCE), Valerius Maximus, Cato, Servius and Dionysius of Halicarnassus [21]. Laurel was abundant, and this can be linked to the origin of the term "Laurentes", which was often used by Virgil and Herodian instead of "Lavinium". Laurel forests in Lavinium were one of the several sacred woodlands on the Roman territory: Varro describes other sacred forests called "lucus Loretae" near the Aventine hill in Rome, and the most famous one near Prima Porta [34,38,66]. Furthermore, considering the historical maps of the site until the XX Century, we could observe the extension of a forested area along the southern coasts of the Latium region [56,67]. However, from these maps, it was not possible to differentiate the various plant communities. 

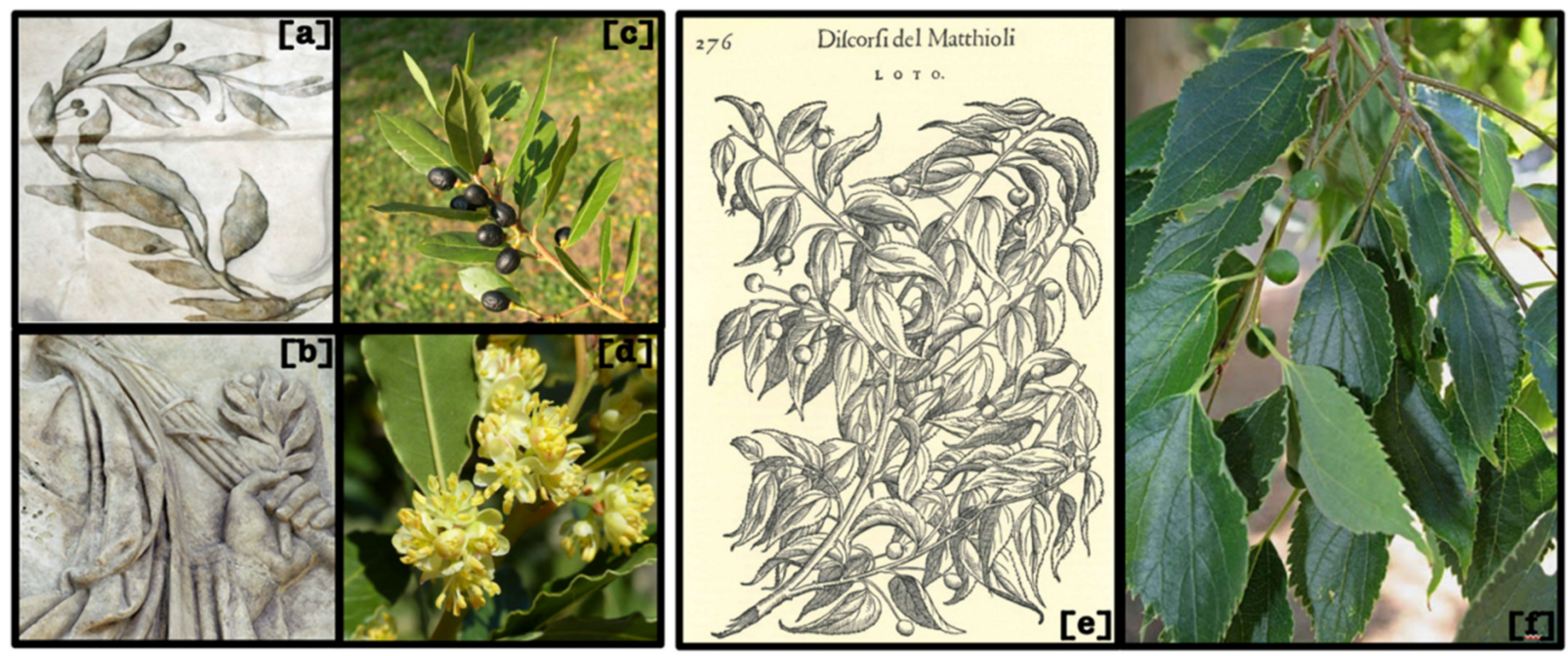

Figure 5. (a,b) Laurel in the Roman iconography, from the carved external front of the Ara Pacis, Rome [40]; (c,d) Details of Laurus nobilis plants; (e) "Lotus" drawing (ancient name for Celtis australis) [45]; (f) Details of C. australis leaves and fruits.

Referring to recent times, laurel trees in the Antica Lavinium SAC show a coppiced habit, linked to specific forest management for wood harvesting. However, this forest exploitation stopped several decades ago, as shown by the absence of logs and cut trees, of clear paths, and the presence of uneven-aged trees both of laurel and other tree species (hackberry, oaks, and ashes), and as reported by Princess Grazia Borghese and by the Estate manager. Specifically, the Princess' effort to conserve the forest and stop any wood exploitation was precedent to the identification of the priority laurel habitat and the establishment of the SAC. The human use of the site is now very limited, considering that the area belongs to a private owner, and visitors can access the area only on sporadic occasions.

\subsection{Linking Man and Nature: Conservation of Natural and Cultural Values}

Among sites in the Latium region, in Antica Lavinium we found an overall good preservation of the forest formations dominated by L. nobilis, but also of the well-integrated mixed formations of L. nobilis with C. australis. These forest conenoses still have certain biodiversity with 128 species of 57 different families [68]. The plant species are mostly Mediterranean, respectively Steno-Mediterranean (34\%) and Euri-Mediterranean (29\%), while European (13\%) and Eurasian species $(15 \%)$ are fewer. Widely distributed species represented only $5 \%$ of the flora and are typical of ecotone areas affected by anthropogenic disturbance (e.g., Stellaria media (L.) Vill., Malva sylvestris L., Urtica dioica L., and Chenopodium album L.). We found a single synanthropic exotic species, Oxalis pes-caprae L., a widespread invasive weed across Mediterranean regions, native to South Africa.

The analysis of the listed species highlights that about $3 \%$ of the analyzed flora is rare or very rare (Fraxinus excelsior L., Quercus petraea (Matt.) Liebl., and Melica minuta L.) at a regional level, while one species (Ruscus aculeatus L.) is listed as an EU Community importance species, and is protected from Regional laws. The analysis of phytogeographic characteristics identified two extrazonal species (Fraxinus excelsior and Sorbus aucuparia L.) and several species as residual elements typical of the Tyrrhenian coast vegetation landscape. In this area, we also noted the great size of some trees and shrubs, such as a specimen of Quercus suber, with a circumference of $5.8 \mathrm{~m}$, Corylus avellana of $5 \mathrm{~m}$ in circumference, C. australis with $2.8 \mathrm{~m}$ and $3.2 \mathrm{~m}$, owing to the low disturbance related to the management of the woodland.

Evaluating the historical interest of the flora, several species were reported as being of particular importance. In fact, almost a third of the species detectable today in the area find traces in Roman iconography (38/128) and these species are: Acanthus mollis, Acer sp., Allium triquetrum, Arisarum vulgare, Arum italicum, Asparagus acutifolius, Bryonia sp., 
Calystegia sylvatica, Campanula sp., Clematis vitalba, Cornus mas, Corylus avellana, Crataegus sp., Cyclamen sp., Cynoglossum creticum, Hedera helix, Humulus lupulus, Laurus nobilis, Ligustrum vulgare, Lychnis flos-cuculi, Malus sylvestris, Malva sp., Olea europaea, Populus nigra, Pteridium aquilinum, Pyrus sp., Quercus cerris, Q. ilex, Q. pubescens, Q. robur, Rosa sp., Salix sp., Silene sp., Smilax aspera, Sonchus sp., Sorbus sp., Symphytum sp., and Vitis vinifera [41,69]. Finally, the ethnobotanical uses of the main investigated trees were reported as still having certain importance for medicinal, aromatic and handicrafts uses. For instance, both L. nobilis and C. australis are still used for their wood in the Latium area to make wooden objects [70].

\section{Discussion}

\subsection{Vegetation Analysis and Comparative Evaluations}

For a more complete comprehension of the dynamics that affected the vegetation formations of the study area, a summary of the historical and ecological evolution of laurel and hackberry populations is needed. Laurel forests were common phytocoenoses in continental Europe during the Tertiary Age, while in the milder periods of the Quaternary Age, they covered wide areas from southern Europe to northern Africa [71]. The laurel forests are now restricted to the Macaronesia, in small and rarefied consortia on the Atlantic coast of the Iberian Peninsula, on the southern coasts of the Black Sea and, more abundantly, in Mediterranean Europe. The Italian peninsula represents one of the major Quaternary refugia of L. nobilis, which can be mostly found in humid and warm sites [13]. Due to the climatic changes that occurred since the Quaternary period, natural laurel forests remained residual in rather small sites in Sardinia, Sicily, and on the Apennines, where they are significantly fragmented and subject to rarefaction $[16,57,72]$. Now, it is a diagnostic species of two habitat types with conservation priority (Annex I of the European Habitat Directive 92/43/CEE, i.e., "5310-Laurus nobilis thickets" and "5230*-Arborescent matorral with Laurus nobilis").

Laurel mostly occupies warm and moist areas with an unmarked seasonality, and it is a shade-tolerant tree, which grows in Mediterranean sclerophyllous communities and on a variety of soil types, avoiding areas with cold winters or a dry climate, preferring relatively humid sites [13]. Furthermore, according to Alessi et al. [12], most of the Italian native laurophylls show a non-equilibrium with the current climate, suggesting that late Quaternary events could explain their current distribution. As such, their presence in central Italy seems to suggest the persistence of a temperate climate in their refugia during Quaternary environmental changes better than ongoing climate changes [12].

The laurel forest vegetation in the Antica Lavinium site seems to be in an ecological transitional area between Mediterranean sclerophyllous, broadleaved woodlands and riparian woods. Such overall heterogeneity is due to the border features of vegetation series' characteristics of this Tyrrhenian sector, i.e., Fraxinus orni-Quercion ilicis, typical of the sclerophyllous Mediterranean vegetation series, and Crataego laevigatae-Quercenion ilicis, characteristic of the Central-Tyrrhenian sub-acidophilic pre-Apennine series [73]. Such communities are also strongly linked to the local geo-morphological and edaphic contexts, i.e., the presence of volcanic soils, ancient dune formations soils, and loamy-clay substrates in the area near to the Fosso di Pratica di Mare. In the latter area, with no anthropic disturbance, the potential vegetation would be the peninsular hygrophilous geosygmetum of riparian vegetation (Salicion albae, Populion albae, Alno-Ulmion), characterized by the dominance, in descending order of hygrophilia, of the genera Salix, Alnus, Fraxinus, Populus and Quercus spp. (Q. robur). Except for alder (Alnus glutinosa), species of the above-listed genera were found (in particular, F. angustifolia subsp. oxycarpa, F. excelsior and F. ornus, together with L. nobilis).

The European hackberry is a species of great relevance too, relict in natural communities that constitutes thermophilous woods in the Mediterranean basin, particularly in North Africa. The optimal growth conditions of $C$. australis include limestone and siliceous grounds, dry and rocky soils lacking in humus, typical of the thermo-Mediterranean and meso-Mediterranean vegetation layers [14]. Today, C. australis mainly grows in north- 
west Africa, southern Europe, Turkey, and western Transcaucasia, while in ancient times, prehistoric evidence has determined its extension to the temperate region of Europe [74] (http:/ / www.plantsoftheworldonline.org, last accessed 12 September 2021). Unfortunately, now woodlands with $C$. australis appear to have been destroyed almost everywhere and are represented only by individual trees and small stands (EUNIS habitat type code G1.7C5; https:/ / eunis.eea.europa.eu/habitats /1165, last accessed 12 September 2021).

The substantial difference between the vegetation with C. australis and L. nobilis, and formations with $L$. nobilis associated with other tree species, which emerged from the PCA analysis (see Figure 3), may be due to the presence of disturbing factors (i.e., water stress, agriculture, grazing, and anthropogenic impacts) that differentiate the vegetation homogeneity along the $Y$-axis in Figure 3. This subgroup, located in the upper section of PCA analysis, includes plots located in the northeast side of the laurel forest formations of the SAC, characterized by closer proximity to the arable fields of the Estate. Plots with the absence of $C$. australis (black dots, Figure 3 ) seem to be arranged on the $X$-axis (of Figure 3 ) according to an increasing mesophilic gradient, so that in the left sector we can find forest species more adapted to warmer and more xeric conditions (e.g., Q. ilex), while in the right sector we can see the presence of more mesophilic and sciaphilous species (e.g., Hedera helix). It is therefore difficult to ascribe these laurel woods to a clearly definite community because the transitionality of the environmental conditions gives rise to variable situations.

In general, results of a phytocoenological classification of $L$. nobilis in Italy showed a wide coenological amplitude of the species with respect to other laurophyllic species, because it grows within a wide range of forest communities [13]. Several associations have been proposed, but their ecological differentiation should be better assessed. The comparative analysis between Antica Lavinium and six other sites from central Italy (Tuscany and Latium) and Italian islands (Sicily and Sardinia) (see Figure 4) showed a wide ecological range of such communities. Our sampling plots are widely arranged in the NMDS graph, reflecting the PCA results, and overlap with the pure laurel forests of the close Castel Porziano, described by Pignatti et al. [30], and of Monte Argentario [29]. The spatial distribution of these forest laurel plots in the PCA described that the main difference between these forests is geographical and could not be ascribed to the presence or absence of the $C$. australis species. In more detail, the left-side cluster includes laurel forests of Central Italy, and the right cluster includes the laurel forests of Sicily and Sardinia, with a further sub-differentiation between forests described in Sardinia (the upper group; Bacchetta et al. [15]) and in Sicily (the lower two groups; Brullo et al. [31] and Gianguzzi et al. [16]). The described mixed laurel-hackberry formations $[15,16,31]$ seem not to show a significant differentiation with the samples when only laurel occurs.

\subsection{Historical and Cultural Analysis of Species and Forests and Their Management}

Historically, both laurel and hackberry were considered trees of high value by Greeks and Romans, but such consideration was progressively lost over time, as often happens for natural elements [50]. In the present times, they are mostly cultivated as ornamental trees and for their aromatic leaves (laurel), but not for their symbolic values [70]. The definition of native populations of $L$. nobilis in Italy sometimes showed certain difficulties, because it is widely cultivated at least since Roman times, and laurel shows a marked attitude to escape from culture and become naturalized [11]. Historical data on Antica Lavinium, from Roman times to modern times, such as the old toponym of Laurentes, can be considered indicators of their native conditions [75].

Hackberry trees, which have a higher mesophilic preference, have often been exploited for their timber. Moreover, during the last 50 years, this species has shown a declining occurrence, especially in urban areas, due to both climate change effects and the action of different pathogens [65]. This species suffers during intense cold and late frosts and droughts, and is attacked by several different phytoplasmas, mites, fungi, and insects [65].

On the ancient coastal Latium region, the evolution of a cultural landscape was characterized by an increase in sclerophyllous taxa, a reduction in the mesophilic forests, 
due to their exploitation, as well as by an expansion of species of agricultural interest, such as Castanea sativa Mill., Juglans regia L. and Vitis sylvestris L. [3,76,77], along with the spread of synanthropic plants (e.g., Urtica, Rumex, and Plantago genera, [6]). Due to the abovementioned cultural and biological value of $L$. nobilis and C. australis, the preservation of such formations in the Antica Lavinium site represents an opportunity for conservation from different viewpoints. It is undoubtable that plant growth in archaeological or cultural sites requires careful planning [20] and spontaneous evolution of vegetation can be harmful for Cultural Heritage conservation [78]. In the case of the Antica Lavinium site, this natural growth does not jeopardize monuments or archaeological remains (mainly located in managed areas outside the forest formations) and becomes, itself, a historical heritage to preserve and maintain.

\subsection{Linking Man and Nature: Conservation of Natural and Cultural Values}

In general, compared to the conditions of the Virgilian mythical places, the city of Lavinium has undergone profound changes over the centuries. They are partly attributable to natural phenomena, such as the progressive changes of the coastline and the variation in the extension of the humid areas behind the dunes, as well as phenomena related to volcanism. However, most changes are largely linked to human actions due to the recent development of a new urban and industrial conglomerate.

Among Latium sites, Antica Lavinium forests still had a relevant floristic and vegetation composition, and noteworthy biodiversity. Both geomorphological and cultural-historical factors seem to have contributed to the conservation of Antica Lavinium laurophyllic forests over the centuries. At first, the geomorphological, climatic, and environmental characteristics of the territory contributed to the conservation of forest communities, because valleys, slopes and peaks affect the local microclimatic conditions and may have reduced the human impact and consequently allowed the preservation of relict taxa and vegetation [9]. With reference to recent management practices, the presence of arable fields, sheep grazing, buffalo farms, and fire have instead promoted the presence of synanthropic species (e.g., Urtica dioica, Stellaria media, Sambucus nigra), counteracting the preservation of communities.

The protected sites of the Natura 2000 network according to the Habitat Directive 92/43/EEC contributed to the maintenance of rare and relict forests and forest priority habitats. The deforestation and forest exploitation were mitigated, as wood harvesting management promoted both the conservation of the relict forests and the co-presence of laurel and other dominant species, creating sub-communities of laurel and oaks, and laurel and hackberries.

Furthermore, mythology, religion, and traditions also played a significant role in landscape management. Several sacred groves were recognized in the Latium region [79], which were protected and preserved because their exploitation was forbidden [80]. The presence of the archaeological remains, together with the management of the Borghese Estate, which preserved the area from urban settlements, also contributed to the conservation of the site.

Furthermore, the cultural, historical, and natural heritage importance of the site was recently (May 2021) reinforced as the site of Antica Lavinium; it was recognized as the final step of "The Aeneas Route" (https:/ / www.aeneasroute.org, last accessed 26 November 2021), among the Cultural Routes of the Council of Europe (https:/ / www.coe.int/en/web/ cultural-routes, last accessed 26 November 2021). Consequently, a careful management plan of the site, valorization and education projects should be planned for the area. This work contributes to highlighting the need to preserve such communities, through the conservation of their habitats.

\section{Conclusions}

Forests dominated by laurel are relict habitats significantly fragmented and subject to rarefaction in the Italian territory. The overall heterogeneity of the forest coenosis of Ancient Lavinium represents a significant, unique case of laurel forests sometimes mixed with hackberry; however, the transitional climatic conditions of the area also make it difficult 
to properly define the community's ecology and syntaxonomy. Such forests and their plants had in the past great importance, and the human impact and management on the landscape over time greatly influenced the plant cover and floristic composition, probably contributing to their conservation, while creating a cultural landscape. The concurrent presence of L. nobilis and C. australis in the site is therefore a result and evidence of the linkage between nature and man, where historical, cultural, and natural aspects mutually contributed to the development of the human and plant communities of this part of the Latium region.

Author Contributions: Conceptualization, G.C.; methodology, G.A.R., F.B., E.C. and F.L.; data analysis, G.A.R., F.B. and E.C.; writing-original draft preparation, G.A.R.; writing-review and editing, G.C., F.B., E.C. and F.L.; supervision, G.C. All authors have read and agreed to the published version of the manuscript.

Funding: Not applicable.

Institutional Review Board Statement: Not applicable.

Informed Consent Statement: Not applicable.

Data Availability Statement: The data presented in this study are available within the paper.

Acknowledgments: The Grant of Excellence Departments, MIUR, Italy (Art. 1, commi 314-337 legge $232 / 2016)$ is gratefully acknowledged.

Conflicts of Interest: The authors declare no conflict of interest.

\section{References}

1. Comes, H.P. The Mediterranean region: A hotspot for plant biogeographic research. New Phytol. 2004, 164, 11-14. [CrossRef]

2. Blondel, J. The 'design' of Mediterranean landscapes: A millennial story of humans and ecological systems during the historic period. Hum. Ecol. 2006, 34, 713-729. [CrossRef]

3. Kelly, M.G.; Huntley, B. An 11 000-year record of vegetation and environment from Lago di Martignano, Latium, Italy. J. Quat. Sci. 1991, 6, 209-224. [CrossRef]

4. Sadori, L.; Giardini, M.; Giraudi, C.; Mazzini, I. The plant landscape of the imperial harbour of Rome. J. Archaeol. Sci. 2010, 37, 3294-3305. [CrossRef]

5. Mensing, S.A.; Schoolman, E.M.; Tunno, I.; Noble, P.J.; Sagnotti, L.; Florindo, F.; Piovesan, G. Historical ecology reveals landscape transformation coincident with cultural development in central Italy since the Roman Period. Sci. Rep. 2018, 8, 1-9. [CrossRef]

6. Sadori, L.; Mercuri, A.M.; Mariotti Lippi, M. Reconstructing past cultural landscape and human impact using pollen and plant macroremains. Plant Biosyst. 2010, 144, 940-951. [CrossRef]

7. UNESCO. Operational Guidelines for the Implementation of the World Heritage Convention; World Heritage Centre: Paris, France, 2019.

8. Hughes, J.D.; Thirgood, J.V. Deforestation, erosion, and forest management in ancient Greece and Rome. J. For. Hist. 1982, 26, 60-75. [CrossRef]

9. Keppel, G.; Van Niel, K.P.; Wardell-Johnson, G.W.; Yates, C.J.; Byrne, M.; Mucina, L.; Franklin, S.E. Refugia: Identifying and understanding safe havens for biodiversity under climate change. Glob. Ecol. Biogeogr. 2012, 21, 393-404. [CrossRef]

10. Le Roux, J.J.; Hui, C.; Castillo, M.L.; Iriondo, J.M.; Keet, J.H.; Khapugin, A.A.; Hirsch, H. Recent anthropogenic plant extinctions differ in biodiversity hotspots and coldspots. Curr. Biol. 2019, 29, 2912-2918. [CrossRef] [PubMed]

11. Filibeck, G. Notes on the distribution of Laurus nobilis L. (Lauraceae) in Italy. Webbia 2006, 61, 45-56. [CrossRef]

12. Alessi, N.; Těšitel, J.; Zerbe, S.; Spada, F.; Agrillo, E.; Wellstein, C. Ancient refugia and present day habitat suitability of native laurophylls in Italy. J. Veg. Sci. 2019, 30, 564-574. [CrossRef]

13. Alessi, N.; Wellstein, C.; Spada, F.; Zerbe, S. Phytocoenological approach to the ecology of Laurus nobilis L. in Italy. Rend. Lincei Sci. Fis. Nat. 2018, 29, 343-354. [CrossRef]

14. Figueiral, I.; Ivorra, S.; Breuil, J.Y.; Bel, V.; Houix, B. Gallo-Roman Nîmes (southern France): A case study on firewood supplies for urban and proto-urban centers (1st BC-3rd AD). Quat. Int. 2017, 458, 103-112. [CrossRef]

15. Bacchetta, G.; Farris, E.; Fenu, G.; Filigheddu, R.; Mattana, E.; Mulè, P. Contributo alla conoscenza dei boschi a Laurus nobilis L. della Sardegna, habitat prioritario ai sensi della Direttiva 92/43/CEE. Fitosociologia 2007, 44, 239-244.

16. Gianguzzi, L.; D’Amico, A.; Romano, S. Phytosociological remarks on residual woodlands of Laurus nobilis in Sicily. Lazaroa 2010, 31, 67. [CrossRef]

17. Marino, P.; Castiglia, G.; Bazan, G.; Domina, G.; Guarino, R. Tertiary relict laurophyll vegetation in the Madonie mountains (Sicily). Acta Bot. Gall. 2014, 161, 47-61. [CrossRef] 
18. Tonelli, V. “Antica Lavinium” Pratica di Mare. In Ambienti di Particolare Interesse Naturalistico del Lazio, Regione Lazio; Dinelli, A., Guarrera, P.M., Eds.; Assessorato alla Cultura, Università La Sapienza, Dipartamente di Biologia Vegetale: Rome, Italy, 1996; pp. 190-192.

19. Caneva, G. A botanical approach to the planning of archaeological, parks in Italy. Conserv. Manag. Archaeol. Sites 1999, 3, 127-134. [CrossRef]

20. Cicinelli, E.; Salerno, G.; Caneva, G. An assessment methodology to combine the preservation of biodiversity and cultural heritage: The San Vincenzo al Volturno historical site (Molise, Italy). Biodivers. Conserv. 2018, 27, 1073-1093. [CrossRef]

21. Castagnoli, F. Lavinium, I. Topografia Generale, Fonti e Storia delle Ricerche; Istituto di Topografia Antica: Roma, Italy, 1972.

22. Blasi, C. Fitoclimatologia del Lazio. Fitosociologia. 1994, 27, 151-175.

23. Jaia, A.M.; Molinari, M.C. Il Santuario di Sol Indiges e il Sistema di Controllo della Costa Laziale nel III sec. a.C. Atti del Convegno VIII di Studi sul Lazio e la Sabina, Roma, 30 Marzo-1 Aprile 2011; Ministero per i Beni e le Attività Culturali, Soprintendenza per i Beni Archeologici del Lazio: Rome, Italy, 2012.

24. Braun-Blanquet, J. Pflanzensoziologie, Grundzüge der Vegetationskunde, 3rd ed.; Springer: Berlin/Heidelberg, Germany, $1964 ;$ p. 631.

25. Pignatti, S.; Guarino, R.; La Rosa, M. Flora d'italia. Edagricole-New Bus. Media 2017, 1, 1120.

26. Bartolucci, F.; Peruzzi, L.; Galasso, G.; Albano, A.; Alessandrini, A.; Ardenghi, N.M.G.; Astuti, G.; Bacchetta, G.; Ballelli, S.; Banfi, E.; et al. An updated checklist of the vascular flora native to Italy. Plant Biosyst. 2018, 152, 179-303. [CrossRef]

27. Braun-Blanquet, J. Plant Sociology. The Study of Plant Communities, 1st ed.; McGraw-Hill: New York, NY, USA, $1932 ;$ p. 539.

28. Lucchese, F.; Pignatti, S. Sguardo sulla vegetazione del Lazio marittimo. In Ricerche Ecologiche, Floristiche e Faunistiche Sulla Fascia Costiera Mediotirrenica Italiana. II. Quaderno n. 264; Accademia Nazionale dei Lincei: Rome, Italy, 1990.

29. Arrigoni, P.V.; Di Tommaso, P.L. La vegetazione del Monte Argentario (Toscana meridionale). Parlatorea 1997, 2, 5-38.

30. Pignatti, E.; Pignatti, S.; D’Angeli, D.; De Nicola, C.; Maffei, L.; Testi, A.; Tinelli, A. The Laurisilva as a cultural heritage: Proposal for the protection of the relict of laurel forest near Ponte Renaro. Rend. Lincei 2015, 26, 643-649. [CrossRef]

31. Brullo, S.; Costanzo, E.; Tomaselli, V. Etude phytosociologique sur les peuplements à Laurus nobilis dans les Monts Iblei (Sicile sud-orientale). Phytocoenologia 2001, 31, 249-270. [CrossRef]

32. Hennekens, S.M.; Schaminee, J.H.J. TURBOVEG Comprehensive database management system for vegetation data. J. Veg. Sci. 2001, 12, 589-591. [CrossRef]

33. Tichy, L. JUICE software for vegetation classification. J. Veg. Sci. 2002, 13, 451-453. [CrossRef]

34. Stara-Tedde, G. I Boschi Sacri dell'Antica Roma. Bull. Comm. Arch. Comunale 1905, 2, 189-232.

35. Baumann, H. Greek Wildflowers and Plant Lore in Ancient Greece; Herbert Press: London, UK, 1993.

36. Grandazzi, A. Lavinium, Alba Longa, Roma: À quoi sert un paysage religieux? Rev. Hist. Relig. 2010, 4, 573-590. [CrossRef]

37. Dierbach, J.H. Flora Mythologica Oder in Bezug auf Mythologie und Symbolik der Griechen und Romer. Ein Beitrag zur a Ltesten Geschichte der Botanik, Agricultur und Medicin; JD Sauerländer: Frankfurt am Main, Germany, 1833.

38. Caneva, G. Ipotesi sul significato simbolico del giardino della Villa di Livia (Prima Porta). Bull. Comm. Archaeol. Comunale Roma 1999, 100, 63-80.

39. Ciarallo, A.M. Elementi Vegetali nell'Iconografia Pompeiana; L'Erma di Bretschneider: Rome, Italy, 2006.

40. Caneva, G. Il Codice Botanico di Augusto: Ara Pacis: Parlare al Popolo Attraverso le Immagini della Natura. The Augustus Botanical Code: Rome, Ara Pacis: Speaking to the People through the Images of Nature; Gangemi: Rome, Italy, 2010.

41. Kumbaric, A.; Caneva, G. Updated outline of floristic richness in Roman iconography. Rend. Lincei Sci. Fis. Nat. 2014, 25, 181-193. [CrossRef]

42. Caneva, G.; Savo, V.; Kumbaric, A. Big Messages in Small Details: Nature in Roman Archaeology. Econ. Bot. 2014, 68, 109-115. [CrossRef]

43. Caneva, G.; Monaco, A.; Virgili, P.; Bartoli, F. "Re-flowering flowers": The hope of an eternal blooming since Roman times. Flora Mediterr. 2019, 29, 27-44. [CrossRef]

44. Caneva, G.; Carpaneto, G.M. Raffaello e l'Immagine della Natura; Silvana Editoriale: Cinisello Balsamo, Italy, 2011.

45. Mattioli, A. I discorsi di M. Pietro Andrea Matthioli, Medico Sanese, nei sei Libri di Pedacio Dioscoride Anazarbeo, della Materia Medica; Libro Co. Italia S.R.L.: San Casciano in Val di pesa, Italy, 2015.

46. Durante, C. Herbario Nuovo; Stamperia Bartolomeo Bonfadino e Tito Diani: Rome, Italy, 1585.

47. Jaia, A.M. Le Mura di Lavinium, Atti delle Giornate di Studio "Le Fortificazioni Arcaiche del Latium Vetus e dell'Etruria Meridionale (IX-VI Sec. a.C.), Stratigrafia, Cronologia e Urbanizzazione"; Academia Belgica, Istituto Storico Belga di Roma: Rome, Italy, 2016.

48. Jaia, A.M.; Molinari, M.C. Two Deposits of Aes Grave from the Sanctuary of Sol Indiges (Torvaianica/Rome): The Dating and Function of the Roman Libral Series. Numis. Chron. 2011, 171, 87-97.

49. Guarrera, P.M. Usi e Tradizioni della Flora Italiana: Medicina Popolare ed Etnobotanica; Aracne: Rome, Italy, 2006.

50. Pardo-de-Santayana, M.; Pieroni, A.; Puri, R. The Ethnobotany of Europe, Past and Present. In Ethnobotany in the New Europe: People, Health and Wild Plant Resources; Pardo-de-Santayana, M., Pieroni, A., Puri, R., Eds.; Berghahn Books: New York, NY, USA, 2010; pp. 1-15.

51. Conti, F.; Manzi, A.; Pedrotti, F. Lista Rossa Nazionale delle Piante d'Italia; W.W.F. \& S.B.I.: Camerino, Italy, 1992.

52. Conti, F.; Manzi, A.; Pedrotti, F. Liste Rosse Regionali delle Piante d'Italia; Dipartimento di Botanica ed Ecologia, Università degli Studi di Camerino: Camerino, Italy, 1997. 
53. Rossi, G.; Montagnani, C.; Gargano, D.; Peruzzi, L.; Abeli, T.; Ravera, S.; Cogoni, A.; Fenu, G.; Magrini, S.; Gennai, M.; et al. Lista Rossa della Flora Italiana. 1. Policy Species e Altre Specie Minacciate; Società Botanica Italiana: Rome, Italy, 2013.

54. Orsenigo, S.; Bacchetta, G.; Calevo, J.; Castello, M.; Cogoni, D.; Gennai, M.; Fenu, G.; Licht, W.; Montagnani, C.; Perrino, E.V.; et al. Global and regional IUCN red list assessments. Ital. Bot. 2018, 9, 111-123. [CrossRef]

55. Orsenigo, S.; Fenu, G.; Gargano, D.; Montagnani, C.; Abeli, T.; Alessandrini, A.; Gianluigi, B.; Fabrizio, B.; Angelino, C.; Rossi, G.; et al. Red list of threatened vascular plants in Italy. Plant Biosys. 2020, 155, 310-335. [CrossRef]

56. Lucchese, F. Atlante della Flora Vascolare del Lazio, Cartografia, Ecologia e Biogeografia. Vol. 2. La Flora di Maggiore Interesse Conservazionistico; Regione Lazio, Direzione Capitale Naturale, Parchi e Aree Protette: Rome, Italy, 2018; p. 400.

57. Allegrezza, M.; Biondi, E.; Felici, S. A phytosociological analysis of the vegetation of the central sector of the Adriatic aspect of the Italian peninsula. Hacquetia 2006, 5, 5-45.

58. Biondi, E. Vegetazione e Habitat Prioritari. Stato della Biodiversità in Italia; MATTM: Rome, Italy, 2005; pp. $202-219$.

59. Biondi, E.; Casavecchia, S.; Pesaresi, S. Direttiva Habitat e conservazione della biodiversità forestale. In Proceedings of the Atti del Terzo Congresso Nazionale di Selvicoltura, Taormina, Italy, 16-19 October 2008.

60. Celesti-Grapow, L.; Alessandrini, A.; Arrigoni, P.V.; Assini, S.; Banfi, E.; Barni, E.; Bovio, M.; Brundu, G.; Cagiotti, M.R.; Camarda, I.; et al. Non-native flora of Italy: Species distribution and threats. Plant Biosyst. 2010, 144, 12-28. [CrossRef]

61. Lucchese, F. Atlante della Flora Alloctona del Lazio: Cartografia, Ecologia e Biogeografia. Vol. 1: Parte Generale e Flora Alloctona. Regione Lazio; Dir. Ambiente e Sistemi Naturali: Rome, Italy, 2017; p. 352.

62. Cristancho, S.; Vining, J. Culturally defined keystone species. Hum. Ecol. Rev. 2004, 11, 153-164.

63. Patrakar, R.; Mansuriya, M.; Patil, P. Phytochemical and pharmacological review on Laurus nobilis. Int. J. Pharm. Chem. Biol. Sci. 2012, 1, 595-602.

64. Allué, E.; Cáceres, I.; Expósito, I.; Canals, A.; Rodríguez, A.; Rosell, J.; Carbonell, E. Celtis remains from the lower pleistocene of Gran Dolina, Atapuerca (Burgos, Spain). J. Archaeol. Sci. 2015, 53, 570-577. [CrossRef]

65. Magni, D.; Caudullo, G. Celtis australis in Europe: Distribution, habitat, usage and threats. In European Atlas of Forest Tree Species, Publication Office of the European Union: Luxembourg, 2016; p. e0145f9.

66. Caneva, G.; Bohuny, L. Botanic analysis of Livia's villa painted flora (Prima Porta, Roma). J. Cult. Herit. 2003, 4, 149-155. [CrossRef]

67. Caneva, G.; Travaglini, C.M. Atlante Storico-Ambientale Anzio e Nettuno; De Luca Editori d'Arte: Rome, Italy, 2003.

68. Albani Rocchetti, G. Aspetti floristico-vegetazionali delle cenosi forestali nell'area dell'Antica Lavinium (Pratica di Mare, Pomezia) nell'ambito della valorizzazione storico-naturalistica dell'area. Not. Soc. Bot. Ital. 2019, 3, 303-338.

69. Jashemski, W.; Meyer, F.G.; Ricciardi, M. Catalogue of plants. In The Natural History of Pompeii; Cambridge University Press: Cambridge, UK, 2002.

70. Guarrera, P.M.; Savo, V.; Caneva, G. Traditional uses of plants in the Tolfa-Cerite-Manziate area (Central Italy). Ethnobiol. Lett. 2015, 6, 119-161. [CrossRef]

71. Rodríguez-Sánchez, F.; Arroyo, J. Reconstructing the demise of Tethyan plants: Climate-driven range dynamics of Laurus since the Pliocene. Glob. Ecol. Biogeogr. 2008, 17, 685-695. [CrossRef]

72. Filibeck, G.; Arrigoni, P.V.; Blasi, C. Some phytogeographical remarks on the forest vegetation of Colchis (Western Georgia). Webbia 2004, 59, 189-214. [CrossRef]

73. Blasi, C.; Di Pietro, R.; Filibeck, G.; Filesi, L.; Ercole, S.; Rosati, L. Le Serie di vegetazione della regione Lazio. In La Vegetazione d'Italia; Palombi \& Partner Srl: Roma, Italy, 2010; pp. 281-310.

74. Simchoni, O.; Kislev, M.E. Early finds of Celtis australis in the southern Levant. Veg. Hist. Archaeobot. 2011, 20, 267. [CrossRef]

75. Blasi, C.; Dowgiallo, G.; Follieri, M.; Lucchese, F.; Magri, D.; Pignatti, S.; Sadori, L. La vegetazione naturale potenziale dell'area romana. In: La vegetazione italiana. Atti Convegni Lincei 1995, 115, 423-457.

76. Allevato, E.; Buonincontri, M.; Vairo, M.; Pecci, A.; Cau, M.A.; Yoneda, M.; Takeuchi, K. Persistence of the cultural landscape in Campania (Southern Italy) before the AD 472 Vesuvius eruption: Archaeoenvironmental data. J. Archaeol. Sci. 2012, 39, 399-406. [CrossRef]

77. Ermolli, E.R.; Romano, P.; Ruello, M.R.; Lumaga, M.R.B. The natural and cultural landscape of Naples (southern Italy) during the Graeco-Roman and Late Antique periods. J. Archaeol. Sci. 2014, 42, 399-411. [CrossRef]

78. Cicinelli, E.; Benelli, F.; Bartoli, F.; Traversetti, L.; Caneva, G. Trends of plant communities growing on the Etruscan tombs (Cerveteri, Italy) related to different management practices. Plant Biosyst. Int. J. Deal. All Asp. Plant Biol. 2020, 154, 158-164. [CrossRef]

79. Ulback, E. The Sacred Groves of Latium and Their Divinities. Class. J. 1934, 29, 658-662.

80. Richardson, L. The cults of Lavinium- Mario Torelli, Lavinio e Roma. Riti iniziatici e matrimonio tra archeologia e storia (Edizioni Quasar, Roma 1984). J. Rom. Archaeol. 1989, 2, 147-150. [CrossRef] 